\title{
Correction to: The effect of intraoperative autofluorescence monitoring on unintentional parathyroid gland excision rates and postoperative PTH concentrations-a single-blind randomized- controlled trial
}

\section{Theodosios S. Papavramidis ${ }^{1,2} \cdot$ Angeliki Chorti $^{1} \cdot$ George Tzikos $^{1} \cdot$ Panagiotis Anagnostis $^{1}{ }^{1} \cdot$ Panteleimon Pantelidis ${ }^{1} \cdot$ Ioannis Pliakos ${ }^{1,2} \cdot$ Stavros Panidis $^{1,2} \cdot$ Maria Papaioannou $^{3} \cdot$ Sohail Bakkar $^{4}$. Ethem Unal ${ }^{5}$. Antonios Michalopoulos ${ }^{1}$}

Published online: 24 May 2021

(c) Springer Science+Business Media, LLC, part of Springer Nature 2021

Correction to: Endocrine

https://doi.org/10.1007/s12020-020-02599-5

The original version of the article has contained a mistake in the following author name.
The author name "Pantelis Pantelidis" has been changed to "Panteleimon Pantelidis"

The original article can be found online at https://doi.org/10.1007/ s12020-020-02599-5.

Panagiotis Anagnostis

pan.anagnostis@gmail.com

$1 \quad$ st Propedeutic Department of Surgery, AHEPA University Hospital, Aristotle University of Thessaloniki, Thessaloniki, Greece

2 Department of Minimal Invasive Endocrine Surgery, Interbalkan Medical Center, Thessaloniki, Greece

3 Laboratory of Biological Chemistry, Faculty of Medicine, School of Health Sciences, Aristotle University of Thessaloniki, Thessaloniki, Greece

4 Faculty of Medicine, Department of Surgery, Hashemite University, Zarqa, Jordan

5 Department of General Surgery, Umraniye Education and Research Hospital, Health Sciences University-Medical Faculty, Istanbul, Turkey 